\title{
Effects of collagen cross-linking on the keratoconus metabolic network
}

\author{
Rabab Sharif ${ }^{1} \cdot$ Henrik Sejersen $^{2} \cdot$ Garrett Frank $^{3} \cdot$ Jesper Hjortdal $\left.\right|^{2} \cdot$ Dimitrios Karamichos ${ }^{1,3}$ \\ Received: 19 October 2017 / Revised: 12 February 2018 / Accepted: 26 February 2018 / Published online: 26 March 2018 \\ (c) The Royal College of Ophthalmologists 2018
}

\begin{abstract}
Purpose Keratoconus (KC) is a multifactorial, ectatic corneal disease. Metabolic changes in the corneal stroma with alterations in collagen fibril stability, oxidative stress, and urea cycle, have previously been reported as key players in KC pathobiology. Recently, corneal collagen cross-linking (CXL) has been introduced as a treatment that can address the progressive nature of $\mathrm{KC}$. While the treatment has been successful in the early days, it is not without clinical ramifications. In this study, we investigated the alterations in KC metabolic profiles due to CXL.

Methods Primary human corneal fibroblasts (HCFs) from healthy donors and human KC fibroblasts (HKCs) from KC donor patients were plated on transwell polycarbonate membranes and stimulated by a stable vitamin C. At 4 weeks, riboflavin was added to the cultures followed by UVA irradiation $(365 \mathrm{~nm})$. Using mass spectrometry, we measured the major differences in metabolites in HKCs compared to HCFs pre- and post CXL.

Result The analysis of 276 metabolites in HCFs and HKCs revealed that the most affected metabolites due to CXL were glutathione disulfide, ascorbic acid, proline, and lysine. A significant decrease in the pro-inflammatory biomarkers (myoinositol and histidine) was also observed. Furthermore, a significant downregulation of many amino acids, lactate levels, and other water-soluble metabolites was noted in HKCs following CXL.

Conclusion CXL is a KC treatment available to patients within certain criteria. Surprisingly, the cellular and molecular mechanisms are considerably understudied limiting our ability for more precise and targeted CXL treatments. In this study, for the first time, we report the effects of CXL on KC metabolism.
\end{abstract}

\section{Introduction}

Keratoconus (KC) is a degenerative corneal disorder, characterized by stromal thinning, protrusion, and corneal scarring. KC prevalence in the general population is 50-200 per 100000 [1]. Central or paracentral corneal stroma endures progressive loss of collagen fibril orientation and biomechanical weakening, initially causing myopia and

$\triangle$ Dimitrios Karamichos

Dimitrios-Karamichos@ouhsc.edu

1 Department of Cell Biology, University of Oklahoma Health Science Center, Oklahoma City, Oklahoma, USA

2 Department of Ophthalmology, Aarhus University Hospital, Aarhus C, Denmark

3 Department of Ophthalmology/Dean McGee Eye Institute, University of Oklahoma Health Science Center, Oklahoma City, Oklahoma, USA irregular astigmatism and eventually leading to significant visual impairment [2, 3]. $\mathrm{KC}$ is usually diagnosed in the patient's adolescent years and is typically detected by corneal topography $[2,4]$, it is progressive until the third to fourth decade of life, when it usually arrests. The reasons for $\mathrm{KC}$ onset, progression, and arrest remain elusive.

Altered tear composition and structural abnormalities in the corneal epithelium, Bowman's layer, and corneal stroma have been suggested as key to the $\mathrm{KC}$ clinical manifestations [5, 6]. It is most commonly an isolated condition, although multiple reports of coexistence with other disorders have been reported [7]. The most recognized systemic and genetic KC associations include Down syndrome, connective tissue disorders, and Leber's congenital amaurosis [1, 7]. $\mathrm{KC}$ is considered a multifactorial disease and is known to result in changes at cellular, physiological, biomechanical, and genetic levels [8]. The main progression characteristic of $\mathrm{KC}$ is a decrease in the biomechanical strength of the cornea, which is 
composed primarily of highly organized collagen and resident keratocytes [9]. Traditionally, KC treatments focus on correcting refractive errors induced by corneal deformation, using spectacles, contact lens, or changing the corneal geometry, as in corneal transplantation [4], which is usually reserved for severe cases. These resources commonly come at a cost and can lead to a number of complications, including allograft reaction, suture and wound-healing problems, and persistent irregular astigmatism [10]. To counter these issues a number of innovations have been introduced at the level of surgical technique, instrumentation, and tissue preparation. However, the disadvantage of these procedures is that none adequately prevent $\mathrm{KC}$ progression that occurs due to the underlying biomechanical corneal changes.

Collagen cross-linking (CXL), introduced in 2008 [11], can prevent the progression of $\mathrm{KC}$ by increasing rigidity of corneal collagens using the chemical production of noncovalent bonds between collagen fibrils. CXL is a technique that uses ultraviolet A (UVA, $365 \mathrm{~nm}$ ) light in combination with riboflavin (vitamin B2) as a photosensitizer. Following exposure, riboflavin is excited into a triplet state thereby producing reactive oxygen species (ROS) [12]. These ROS further act to induce the formation of new covalent bonds between the amino acids of the adjacent collagen fibers thereby improving corneal biomechanical resistance and stability [13]. Spoerl et al. [14] were the first to study the effects of riboflavin/UVA therapy in the cornea, to achieve CXL. Using this technique, important positive effects of CXL on the properties of both human and porcine corneal tissue were demonstrated by Wollensak et al. [15] and Raiskup-Wolf et al. [16]. Various clinical studies have since shown that CXL slows and in most cases blocks KC progression [17-19]. The riboflavin also offers protection to the deeper ocular structures, such as endothelium, lens, and retina, by absorbing the UVA [20]. Unfortunately, not every KC patient will qualify for CXL [12].

With recent advances in mass spectrometry over the last few years, there has been increasing interest in exploring potential metabolites that are associated with KC pathogenesis. To the best of our knowledge, our group was the first to test metabolomics in $\mathrm{KC}$, utilizing human tears, saliva, and cells from $\mathrm{KC}$ donors, allowing the simultaneous analysis of hundreds of metabolites [6, 21-24].

In the current study, we investigated the metabolic effects pre/post CXL in KC-derived corneal cells, using our three-dimensional (3D) in vitro model. Our data reveal an otherwise unknown CXL mechanism of action in regulating corneal bioenergetics. This study highlights the actions of CXL and delineates a critical aspect of this clinical treatment.

\section{Materials and methods}

\section{Ethics}

All procedures used in these studies adhered to the tenets of the Declaration of Helsinki. KC donor corneas were obtained from Drs. Hjortdal (Aarhus University Hospital, Aarhus, Denmark), and Frank (Dean McGee Eye Institute, USA). Institutional review board (IRB) approval was received prior to initiation of experiments described in this study both at Aarhus University Hospital and Dean McGee Eye Institute (\#3450). Written consent was obtained prior to sample collection. Inclusion/exclusion criteria for data collection were established prior to the onset of sample collection. Inclusion criteria for healthy controls required absence of $\mathrm{KC}$ diagnosis or any other corneal diseases or atopic disorders. Inclusion criteria for $\mathrm{KC}$ patients required diagnosis of $\mathrm{KC}$ by a certified ophthalmologist and absence of other ophthalmic conditions, or patients who had previously received CXL or undergone penetrating keratoplasty.

\section{Cell isolation}

Healthy and KC human corneas were obtained and processed as previously described [25, 26]. Donor information for HCFs utilized in this study: N21 (63-year-old (y/o) male); N22 (69 y/o male); N23 (21 y/o male); and N24 (43 y/o female). Donor information for HKCs utilized in this study: WU2 (69 y/o male); WU3 (34 y/o male); DM1 (44 y/o female); and GF2 (53 y/o female). Through brief scraping with a razor blade, the endothelium and epithelium were removed from the stroma. The stromal tissue was then cut into small pieces $(\sim 2 \mathrm{~mm} \times 2 \mathrm{~mm})$. Tissue was then allowed to adhere to the bottom of a T25 flask for $30 \mathrm{~min}$ at $37^{\circ} \mathrm{C}$ before carefully adding Eagle's minimum essential media (EMEM; American Type Culture Collection, Manassas, VA) containing 10\% fetal bovine serum (FBS; Atlantic Biologic's, Lawrenceville, CA) and $1 \%$ antibiotic (Gibco Antibiotic-Antimycotic, Life Technologies) to the flask without disturbing the explants. At approximately $100 \%$ confluency, explants were further passaged into $\mathrm{T} 75$ flask and incubated at $37^{\circ} \mathrm{C}, 5 \% \mathrm{CO} 2$ for further expansion.

\section{D cell cultures and extracellular matrix assembly}

Primary human corneal fibroblasts (HCFs) from healthy patients and human $\mathrm{KC}$ fibroblasts (HKCs) from $\mathrm{KC}$ donor patients were seeded on a transwell six-well plates with polycarbonate membrane inserts with $0.4-\mu \mathrm{m}$ pores (Transwell; Corning Costar, Charlotte, NC) at a density of $1 \times 10^{6}$ cells/well and cultured in a 10\% FBS EMEM medium and $1 \%$ antibiotic, stimulated with $0.5 \mathrm{mM} 2-O-\alpha-\mathrm{D}-$ glucopyranosyl-L-ascorbic acid (vitamin C, American Custom 
Chemicals Corporation, San Diego, CA). Cultures were grown for 4 weeks before further processing, and fresh media was supplied every other day for the duration of the experiments [24, 26].

\section{Corneal CXL}

We have recently developed our 3D in vitro model to accommodate riboflavin-UVA-CXL, establishing a setup mimicking the current clinical treatment of KC. In this model, both cell types (HCFs and HKCs) were plated on transwell polycarbonate membrane inserts, at the end of week 4 , a mixed riboflavin $0.1 \%$ phosphate-buffered saline (PBS) solution was added to the constructs followed by UVA irradiation to ensure riboflavin cell saturation. A UV-X illumination device (v.1000;IROC AG, Zurich, Switzerland) was used at a wavelength of $370 \mathrm{~nm}$ and an irradiance of $3 \mathrm{~mW} / \mathrm{cm}^{2}$ of UVA. The UV-X device was calibrated prior to each treatment using a UVA meter (LaserMate-Q; LASER 2000, Wessling, Germany), each well was exposed to UVA for $3 \mathrm{~min}$ at a $3 \mathrm{~cm}$ distance, mirroring current CXL clinical settings. Post irradiation, each construct was rinsed with PBS and incubated in fresh media for 12-24 $\mathrm{h}$ before further analysis [27].

\section{Metabolite extraction}

As previously reported [6, 21-23, 28], metabolites were isolated from the $3 \mathrm{D}$ in vitro cultures using ice-cold $80 \%$ $\mathrm{MeOH}$ centrifuged $\left(14000 \times g, 10 \mathrm{~min}, 4^{\circ} \mathrm{C}\right)$ and supernatants incubated on dry ice. Pellets were further disrupted in ice-cold $80 \% \mathrm{MeOH}$ and combined with previous supernatants. Plasma metabolites were extracted twice in $80 \%$ ice-cold $\mathrm{MeOH}$. Metabolite extracts were vortexed and centrifuged $\left(14000 \times g, 10 \mathrm{~min}, 4^{\circ} \mathrm{C}\right)$. Supernatants were evaporated and stored at $-80^{\circ} \mathrm{C}$ until further analysis.

\section{Targeted mass spectrometry}

As previously described [28], extracted metabolites were isolated using ice-cold $\left(-80^{\circ} \mathrm{C}\right) \mathrm{MeOH}$ in water on dry ice, incubated, centrifuged, dried, and repeated two times. Dried pellets were processed for metabolomics analysis. Highperformance liquid chromatography-grade water was used to dissolve pellets and analyzed for quantification by Liquid chromatography tandem-mass spectrometry (LC-MS/MS) in a hybrid 5500 QTRAP triple quadrupole mass spectrometer (AB/SCIEX) [6, 21, 23, 24, 29].

\section{Pathway enrichment analysis}

Pathway enrichment analysis was performed using online software; MetaboAnalyst (www.Metaboanalyst.ca) intended for the analysis of metabolomics data [28]. Briefly, we performed the analysis using only the metabolites that were up- or downregulated by at least twofold as indicated by our statistical analysis. Only metabolites that were present on all biological samples were considered for further analysis. The metabolites that passed our criteria were input into the software and the pathway enrichment analysis was performed [28]. The output of this algorithm marks a metabolic pathway if the input list of the metabolites suggests that the pathway associated with a specific pathway are present at levels that would not be expected by random chance.

\section{Statistical analysis}

A two-way analysis of variance with multiple comparison analysis based on Tukey's method was performed in GraphPad Prism to determine significance with $p<$ 0.05 considered statistically significant. Randomized allocation and blinded analysis of human samples was applied for the duration of the executed experiments. The $n$ number for each experiment is listed in the appropriate figure legend, and all were performed in triplicate. All graphs show mean \pm standard error of the mean. Sample size was chosen following consultation with a biostatistician on campus. Briefly, our experiments showed low variability and the differences between conditions were significant enough so that the chosen $n$ numbers are statistically valid and according to our power analysis.

\section{Results}

\section{CXL: metabolic enriched pathways}

$\mathrm{KC}$ is known to be a disease of multifactorial origin. Metabolic disorders, involving changes in the urea cycle, alteration in the oxidative stress level, or changes in hydroxylated proteins, have been reported to influence the development of $\mathrm{KC}$ [2]. In this study we investigated the differences in metabolic profile of HKCs and HCFs pre- and post CXL, in order to determine if CXL regulates cellular metabolism. Four groups were included and analyzed: (1) HCF controls (pre-CXL), HCF.c; (2) HKC controls (pre-CXL), HKC.c; (3) HCF CXL (post CXL), HCF.x; and (4) HKC CXL (post CXL), HKC.X.

The primary pathways enriched (Fig. 1) in HCF.x and HKC.x involved methionine metabolism, betaine metabolism, protein biosynthesis (threonine, adenosine, cysteine, and tyrosine metabolism), glycine and threonine metabolism, in addition to collagen intermediate residues (proline and lysine; Fig. 1a). Metabolites critical in cell 
(A)

Metabolite Sets Enrichment Overview

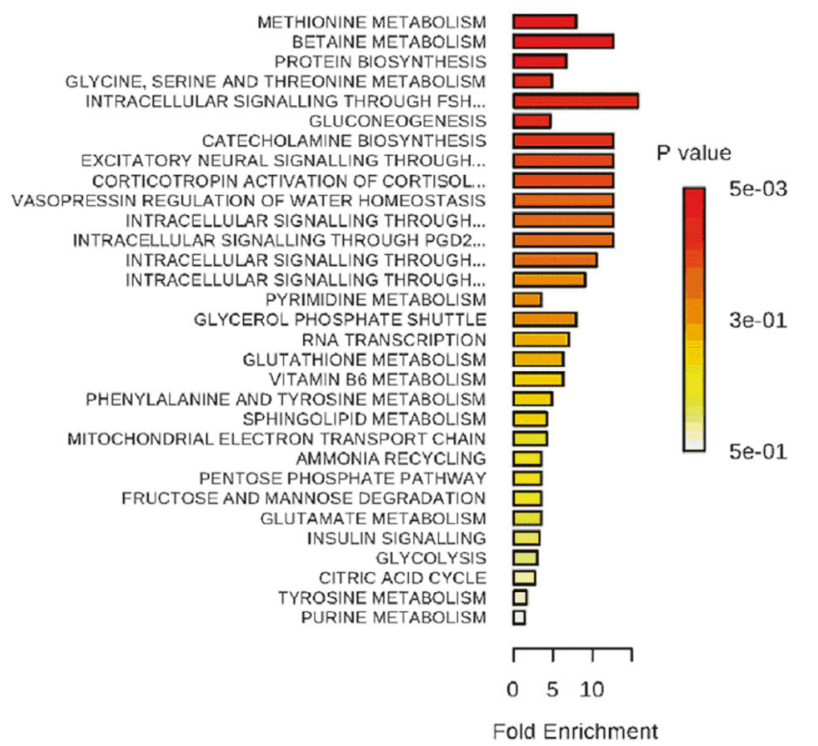

(B)

Metabolite Sets Enrichment Overview

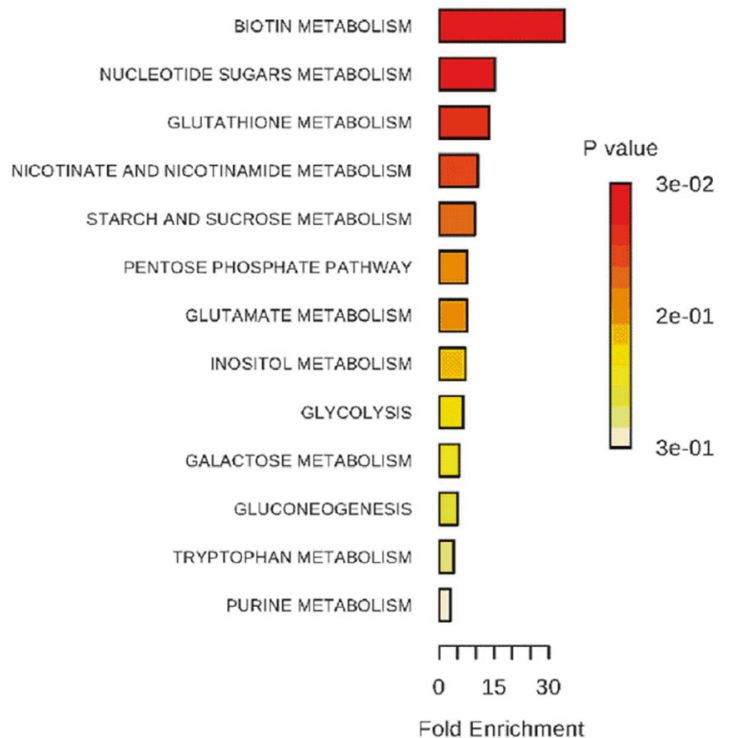

(C)

Metabolite Sets Enrichment Overview

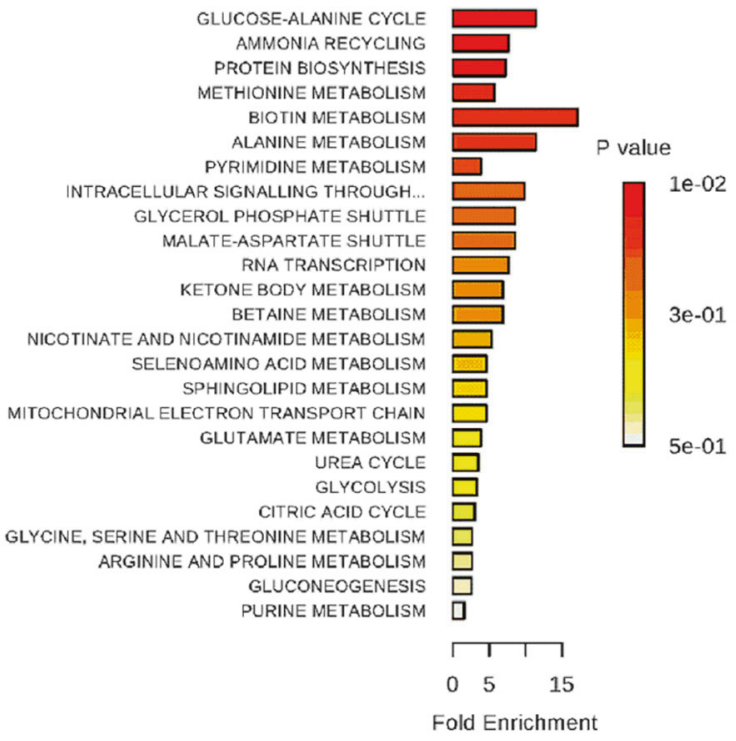

Fig.1 Enrichment pathways identified in HCFs and HKCs by MetaboAnalyst following CXL treatment. a Effects of CXL on HCF.c vs
HCF.x. b Effects of CXL on HKC.c vs HKC.x. c Effects of CXL on HCF.c vs HCF.x

\section{CXL: oxidative stress}

Oxidative stress-related pathways were significantly affected and are shown in Fig. 2. A variety of antioxidant enzymes are present in the cornea such as catalase, GSH peroxidase, and GSH reductase (GR), ensuring the removal of ROS generated by UV light exposure [30]. Cells will also defend themselves with enzymes such as superoxide dismutase (SOD) and GSH peroxidases [24]. Small- 
(A)

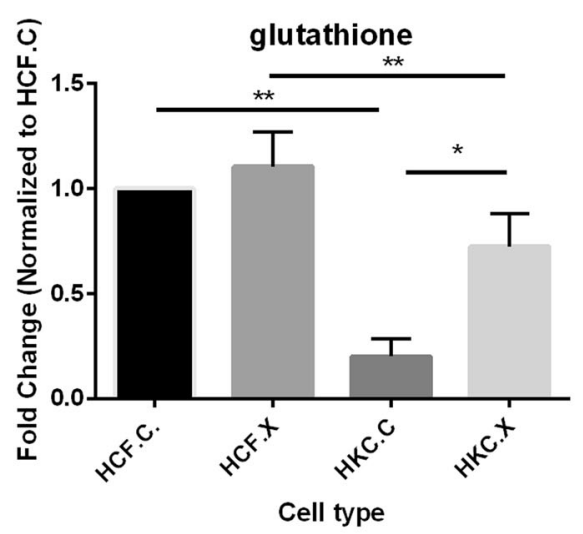

(C)

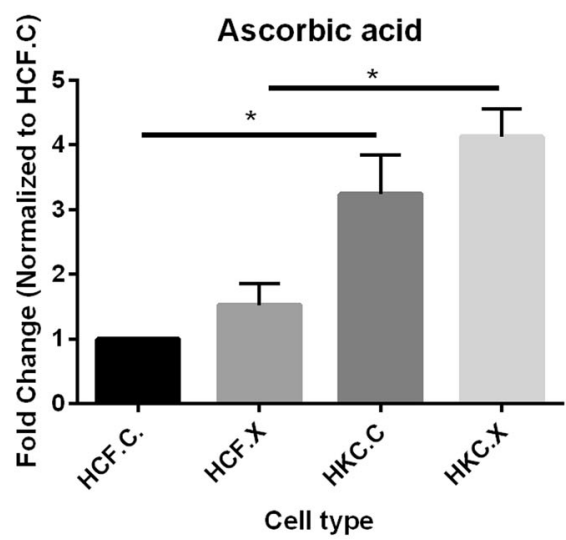

(B)

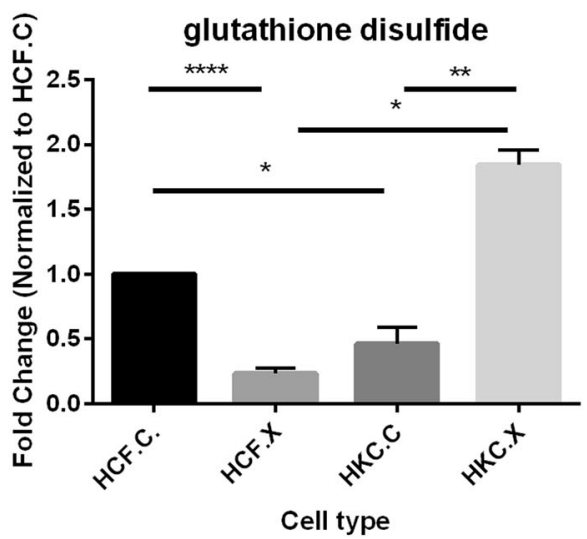

(D)

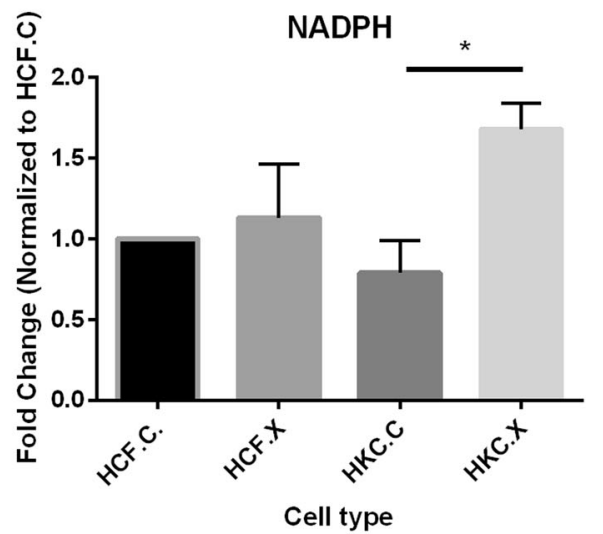

(E)

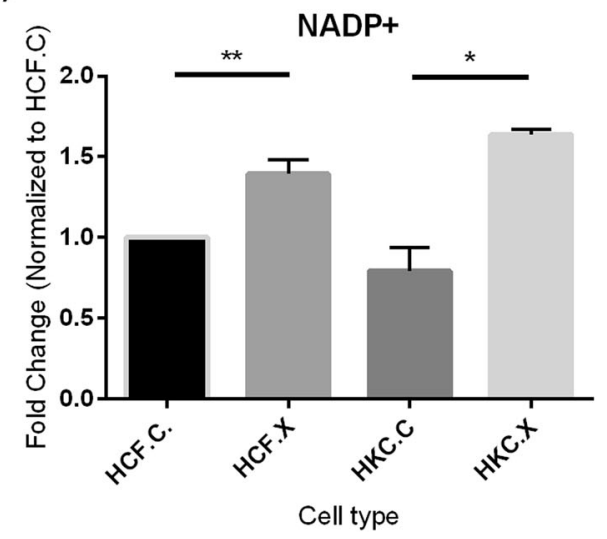

Fig.2 Quantitative analysis of metabolite levels identified by targeted LC-MS/MS in corneal cell lysates isolated from healthy controls ( $\mathrm{HCFs}$ ) and keratoconus patients (HKCs), revealing the effects of CXL

molecule antioxidants such as GSH and ascorbic acid also play a vital role in the cornea defense mechanism. GSH is ubiquitously synthesized in all cell types. It is an antioxidant tripeptide comprising glutamate, cysteine and glycine. As the most abundant cellular non-protein thiol, the ability of reduced GSH to convert to its oxidized form (GSH on glutathione metabolism. a Glutathione, $\mathbf{b}$ glutathione disulfide, $\mathbf{c}$ ascorbic acid, $\mathbf{d} \mathrm{NADPH}$, and e NADP $+. n=4, * p<0.05, * * p<0.01$, $* * * * p<0.0001$

disulfide; GSSH) depending on the oxidative milieu allows it to function as a major cellular redox buffer. In our study, HKC.x show a significant increase in GSH and GSSG (Fig. 2a, b; $p<0.05$ and $<0.01$, respectively) compared to HKC.c. Under conditions of oxidative stress, fast turnover of corneal GSH via this pathway, in combination with 
efficient recycling by GR, are required to counteract free radicals [31]. GSH also plays a pivotal role in maintaining optimum hydration of the cornea, protecting the integrity of its cellular membranes [7].

Our data also showed upregulation of ascorbic acid in HKC.x compared to HCF.x (Fig. 2c; $p<0.05$ ). While ascorbic acid is known to promote healing of damaged corneal tissues by serving as an essential cofactor for collagen synthesis in corneal fibroblasts, corneal ascorbic acid is also believed to be the most important non-enzymatic antioxidant in the cornea [31-34].

In addition to GSH, ascorbic acid, and uric acid, other small molecules also contribute to the overall antioxidant capacity within ocular tissues. The activity of extracellular SOD and content of GSH are decreased in KC corneas in comparison to the normal samples. We determined the levels of nicotinamide adenine dinucleotide phosphate NADPH and its oxidized product, nicotinamide adenine dinucleotide phosphate (NADP+), together have the primary function of being an important cellular redox buffer. Corneal NADPH has been reported to act as an antioxidant through several mechanisms [35]. HKC.x showed a slight increase in NADPH and its oxidized product, NADP+ (Fig. 2d, e; $p<0.05$ ).

\section{CXL: citric acid cycle and glucose}

$\mathrm{KC}$ is known to be associated with altered cellular metabolism involving the citric acid cycle and increased lactate production [24, 29]. A significant decrease in lactate levels was observed in HKC.x when compared to HKC.c (Fig. 3a; $p<0.01)$. In addition, CXL led to a significant increase in ATP observed in HKC.x compared to HCF.x and HKC.c (Fig. 3b; $p<0.01$ and $<0.001$, respectively). These results suggest that CXL increased the ability of these cells to produce high-energy phosphate compounds crucial for cellular function.

Furthermore, CXL led to a significant decrease in orotate $(p<0.01)$ and adenosine levels $(p<0.05)$ in HKC.x compared to HKC.c (Fig. 3c, d). However, a significant increase in dAMP levels in HKC.x compared to HKC.c (Fig. 3e; $p<0.01$ ), and both hexose-phosphate levels (Fig. 3f; $p<0.05$ ) and glucose 6-phosphate (Fig. 3g; $p<0.05$ ) in HKC.x compared to HKC.c was observed, suggesting that CXL may contribute to altered energy production by affecting nucleotide availability and glucose metabolism in $\mathrm{KC}$.

\section{CXL: amino acids and collagen}

The amino acid tyrosine is electrochemically active and scavenges hydroxyl radicals and singlet oxygen species [36, 37]. Also, cysteine replenishes the tissue GSH pool by replenishing the rate-limiting substrate for GSH biosynthesis and acting as a direct antioxidant via its thiol group [32, 38]. Our data show that CXL results in a significant increase in both cysteine and tyrosine in HKC.x compared to HKC.c (Fig. $4 \mathrm{a} ; p<0.01$ and $4 \mathrm{~b} ; p<0.05$ ). Such an antioxidant defense system in the ocular surface is essential for the maintenance of redox homeostasis in the eye and protection against oxidative damage [37].

One of the critical factors for the structural and biomechanical functions of collagen is the post-translational modifications (PTMs), including, hydroxylation and glycosylation of peptidyl lysine and proline residues, procollagen processing, and progressive CXL [39]. In KC a majority of collagens show reduced hydroxyprolinecontaining peptides, contributing to collagen structural abnormalities and biomechanical weakness as seen in $\mathrm{KC}$.

Moreover, we analyzed the presence of collagen intermediates (proline and lysine) that undergo various PTMs. Our results show a significant increase in lysine and proline (Fig. 4c, d; $p<0.01$ ) in HKC.x compared to HKC.c, this potentially indicates that abundant collagen intermediates are available to undergo PTMs and ensure proper collagen synthesis and assembly. This suggests that CXL plays a key role in regulating and orchestrating the formation of a wellassembled extracellular matrix.

In addition, pro-inflammatory metabolites such as myoinositol and histidine are significantly downregulated in HKC.x compared to HKC.c (Fig. $4 \mathrm{e}$, f; $p<0.01$ ).

\section{CXL: lipid biosynthesis}

Cholesterol serves as a precursor to steroid biosynthesis, as well as an essential structural component of the lipid bilayer [40]. Cholesterol sulfate also functions as a source of steroid precursors, and is a regulator of phosphatidylinositol 3kinase and protein kinase $C$ [29]. No change was found in basal cholesterol levels (Fig. 5a) but a decrease in cholesterol sulfate in HKC.x was observed compared to HKC.c and HCF.x (Fig. 5b; $p<0.01$ ).

Glycerophosphocholine (Fig. 5c), glycerol-3-phosphate (Fig. 5d), and 1,3-diphosphateglycerate (Fig. 5e), important mediators involved in lipid signaling and lipogenesis, were decreased in HKC.x compared to HKC.c $(p<0.01,<0.01$, and $<0.05$, respectively).

\section{Discussion}

Several studies have suggested that oxidative stress may be involved in $\mathrm{KC}$ [41]. It is widely known that the accumulation of ROS can damage cells by reacting with DNA, membrane phospholipids, and proteins [36]. Normally, the 
Fig.3 Quantitative analysis of metabolite levels identified by targeted LC-MS/MS in corneal cell lysates isolated from healthy controls (HCFs) and

keratoconus patients (HKCs), revealing the effects of CXL on glucose metabolism and citric acid cycle metabolites. a

Lactate, b ATP, c orotate, d adenosine, e dAMP, f hexose phosphate, and g glucose 6phosphate. $n=4, * p<0.05$, $* * p<0.01, * * * * p<0.0001$
(A)

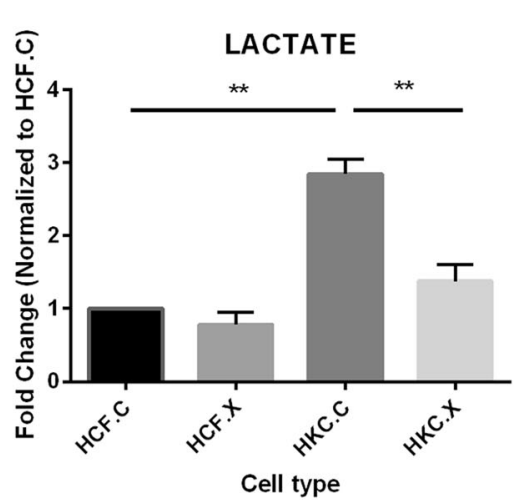

(C)

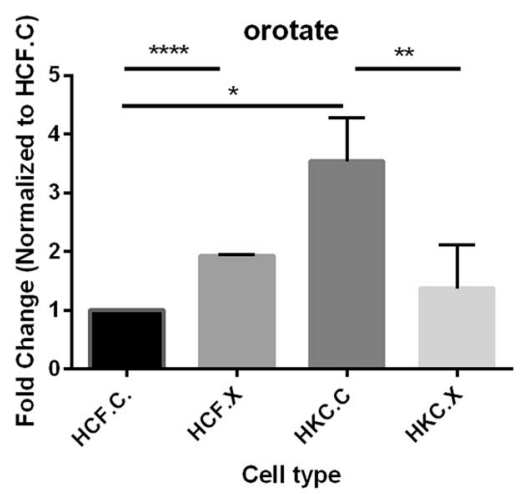

(E)

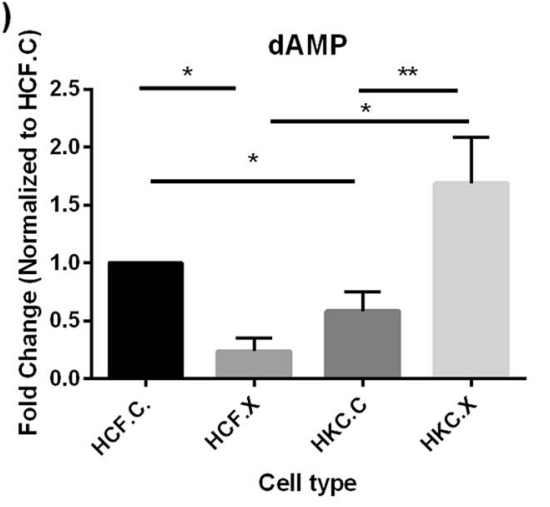

(B)

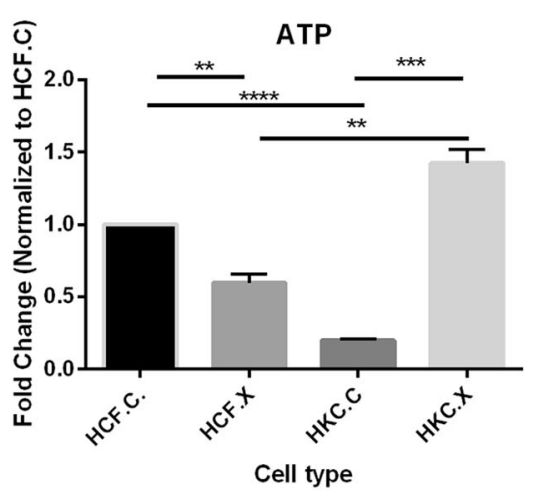

(D)

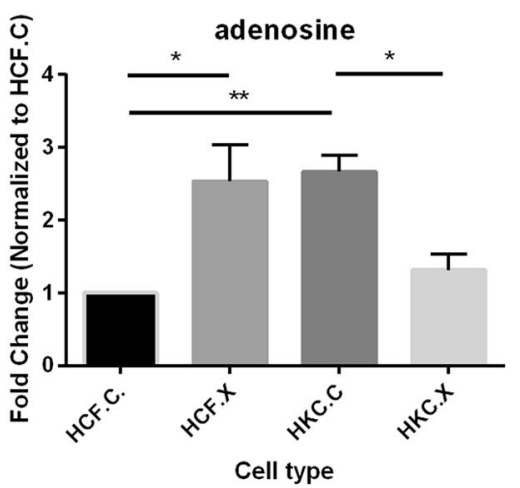

(F)

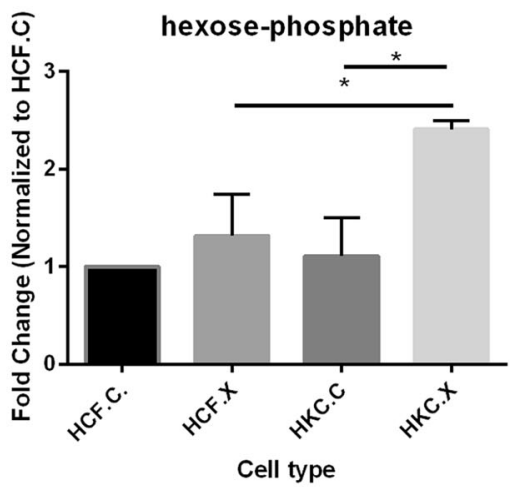

(G)

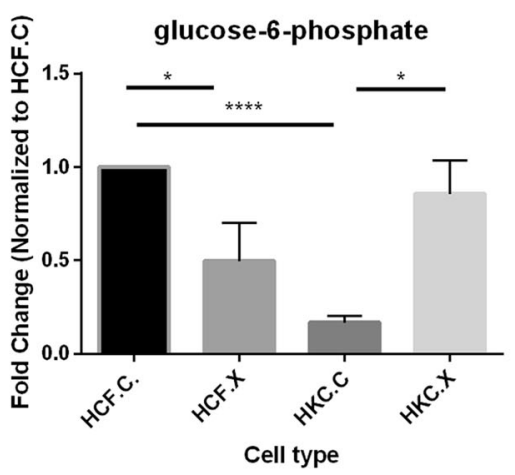


(A)

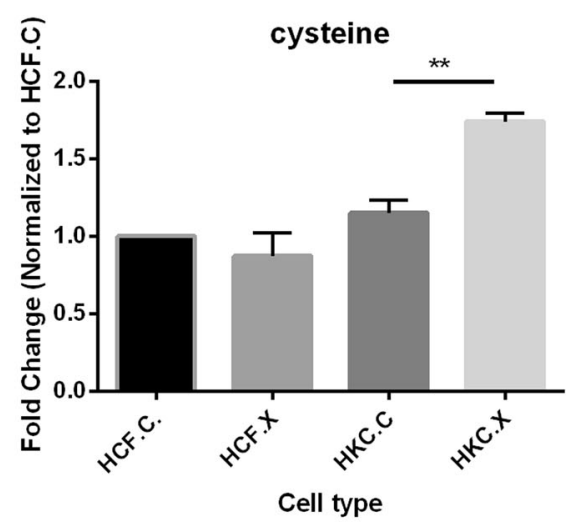

(C)

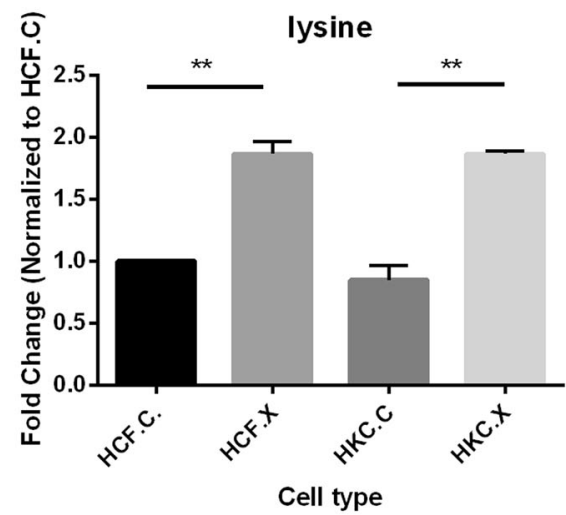

(E)

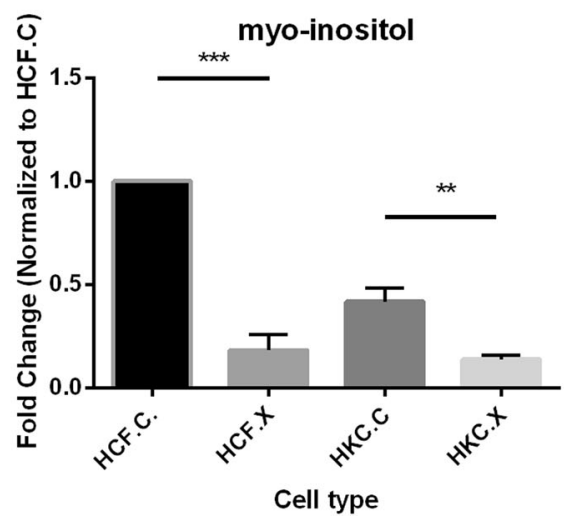

Fig.4 Quantitative analysis of metabolite levels identified by targeted LC-MS/MS in corneal cell lysates isolated from healthy controls (HCFs) and keratoconus patients (HKCs), revealing the effects of CXL

cornea's natural antioxidant enzymes eliminate the ROS before they damage cells; these include SOD, GR, catalase, and GSH peroxidase [23]. However, in the disease state, such as KC, ROS seems to overcome cellular defenses and promote cell damage [42]. Cellular damage can also be promoted by various products formed during ROS-induced lipid peroxidation [43] and the release of different reactive aldehydes, such as 4-hydroxy-2, 3-trans-nonenal (HNE) [30]. The HNE can modify proteins on cysteine, lysine, and
(B)

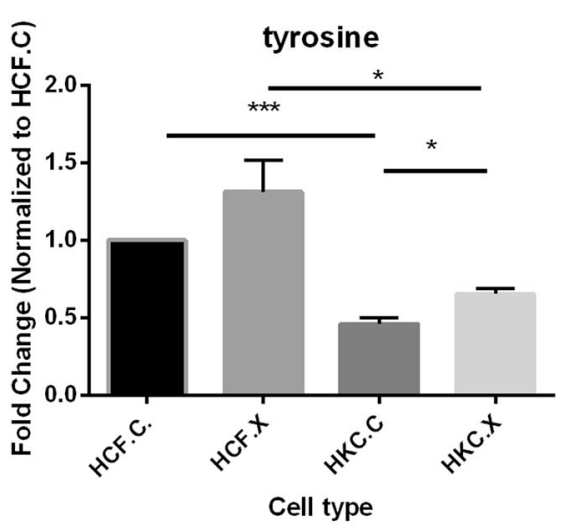

(D)

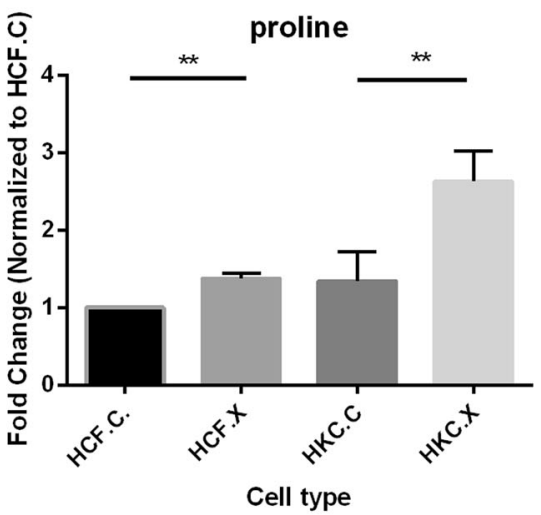

(F)

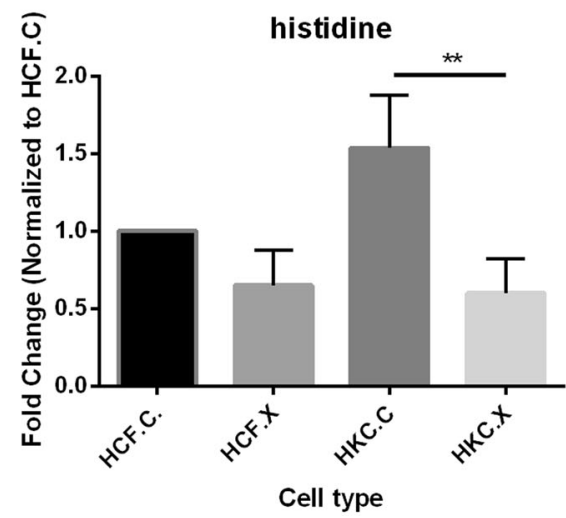

on amino acids, and collagen intermediates. a Cysteine, b tyrosine, $\mathbf{c}$ lysine, d proline, e myo-inositol, and f histidine. $n=4, * p<0.05$, $* * p<0.01, * * * p<0.001$

histidine residues and such modifications can impair protein function and promote protein aggregation [38].

Although some antioxidant enzymatic activities and protein levels have been examined in normal and $\mathrm{KC}$ corneas [20, 29], the role of oxidative stress and HNE in $\mathrm{KC}$ corneas is unclear. Through various mechanisms, cells attempt to remove stressors, decrease damage, and maintain homeostasis. However, various internal deleterious changes can happen during this process [2]. Many proteins 
(A)

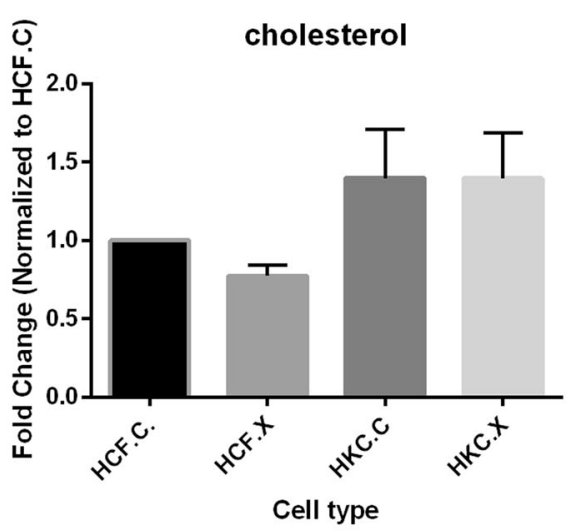

(C)

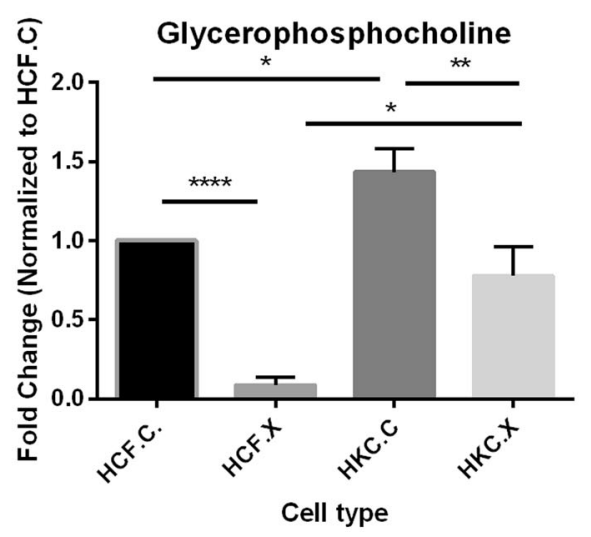

(B)

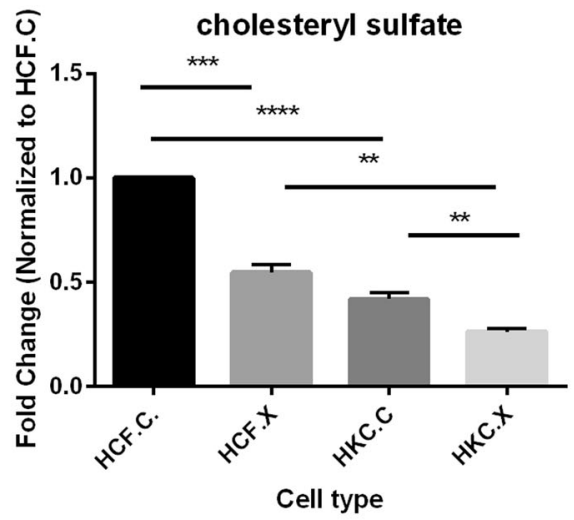

(D)

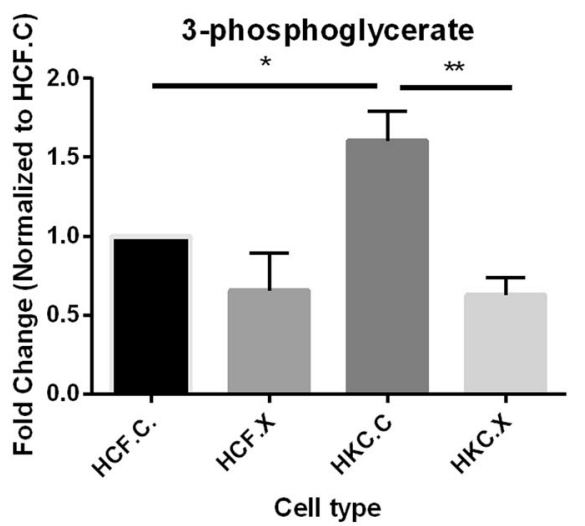

(E)

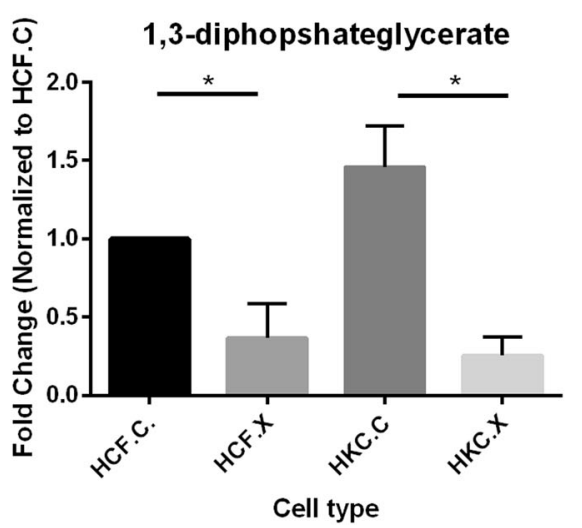

Fig.5 Quantitative analysis of metabolite levels identified by targeted LC-MS/MS in corneal cell lysates isolated from healthy controls (HCFs) and keratoconus patients (HKCs), revealing the effects of CXL on lipid biosynthesis. a Cholesterol, b cholesteryl sulfate,

that are involved in free radical detoxification, such as GHS, catalase, SOD, and superoxide GSH, have been shown to have decreased activity in KC [44]. Thus, alterations in ROS following CXL reported herein are novel and may provide new insights into the pathophysiology of KC. It also highlights a somewhat protective role of CXL. c glycerophosphocholine, d 3-phosphoglycate, and e 1,3-diphosphateglycerate. $\quad n=4, \quad * p<0.05, \quad * * p<0.01, \quad * * * p<0.001$, $* * * * p<0.0001$

Although ROS is critical for physiological functions, including cell growth, proliferation, differentiation, and apoptosis, their excess may have a detrimental influence on cellular components [24]. They can cause oxidative modifications of biomolecules, including lipids, proteins, carbohydrates, and nucleic acids, leading to cellular and tissue damage.The cornea is affected not only by the accumulation 
of ROS [24, 36] but also via the malfunctions of corneal antioxidant defense mechanisms, leading to structural and functional changes in this tissue. As a result, mitochondria can be severely affected. Mitochondria are the major site of oxygen metabolism, they are the most important source of endogenous production of ROS [45]. DNA damage induced by oxidative stress may affect the protein-coding region of mitochondrial DNA and influence oxidative phosphorylation [3]. Changes in the expression of oxidative phosphorylation proteins may lead to improper ATP synthesis, increased ROS formation, and more oxidative damage [41]. An association between mitochondrial dysfunction and a variety of diseases has been shown $[30,46]$, and it is now linked to KC progression as well [29]. In our study, we observed changes in critical metabolites such as adenosine, cysteine, threonine, and ascorbate. An increase in ascorbate concentration after CXL suggests its potential to reduce the amount of corneal damage and promote wound healing. CXL induced significant reductions in adenosine, lactate, and GSH while levels of glucose were elevated, and correlated with a decrease in lactate levels. Based on our results in this study, CXL seems to have a positive impact on KC microenvironment. We showed that CXL can impact different metabolic processes, such as antioxidant changes and lactate with inflammation, the changes in the hydroxylated products of CXL, and amino acid levels. These alterations following CXL could potentially be a CXL time-dependent effect on KC metabolic profile. Further long-term studies will help shed the light on the metabolic consequences of the disease, and improve CXL therapeutic techniques, potentially eliminating undesired short- and long-term complications.

Using a human $\mathrm{KC}$ in vitro model, we take the lead in examining the influence of CXL on the metabolic profile of $\mathrm{KC}$ and healthy donors. Moreover, metabolomics provides a profile of pathways and processes that have been altered in KC following CXL. The results presented in this study strongly support further investigation of metabolic analysis of KC following different CXL time points, which could potentially highlight the main aim of metabolomics in identifying, measuring, and interpreting the complex timerelated concentration and activity of endogenous metabolites in cells and tissues. This leads to a greater understanding of the disease condition and underlying mechanisms involved. The advancement of experimental techniques in the field of metabolomics has provided a good understanding of the metabolism of normal as well as diseased ocular tissue and treatment modalities. Unique metabolic profile differences identified through this technique are valuable in aiding future diagnosis of $\mathrm{KC}$ and serve as predictors of disease progression as well as CXL treatment complications and outcome.

\section{Summary}

\section{What was known before}

- Keratoconus (KC) is a multifactorial, non-inflammatory disease of the human cornea.

- Corneal collagen cross-linking (CXL) has been introduced as a $\mathrm{KC}$ treatment that can address the progressive nature of the disease.

- Cellular and molecular mechanisms are considerably understudied.

\section{What this study adds}

- For the first time, we report the effects of CXL on KC metabolism.

Acknowledgements We acknowledge the use of tissues procured by the National Disease Research Interchange (NDRI) with support from NIH grant U42OD11158. The authors would like to thank Drs. John Asara and Min Yuan for technical assistance with metabolomics experiments. The authors also thank our biostatistician, Dr. Ding Kai.

Funding The authors would like to acknowledge the support of the National Institute of Health (NEI) Grants EY023568 (DK). This work was supported by an unrestricted grant (DMEI) from Research to Prevent Blindness (New York, NY USA). The funders have no role in study design, data collection and analysis, decision to publish, or preparation of the manuscript.

\section{Compliance with ethical standards}

Conflict of interest The authors declare that they have no conflict of interest.

\section{References}

1. Sherwin T, Brookes NH. Morphological changes in keratoconus: pathology or pathogenesis. Clin Exp Ophthalmol. 2004;32:211-7.

2. Davidson AE, Hayes S, Hardcastle AJ, Tuft SJ. The pathogenesis of keratoconus. Eye. 2014;28:189-95.

3. Ionescu C, Corbu CG, Tanase C, Jonescu-Cuypers C, Nicula C, Dascalescu D, et al. Inflammatory biomarkers profile as microenvironmental expression in keratoconus. Dis Markers. 2016;2016:8.

4. Amanzadeh K, Karimian F, Khanlari M. Management of keratoconus. J Ophthalmic Vis Res. 2012;7:341-6.

5. Young SP, Wallace GR. Metabolomic analysis of human disease and its application to the eye. $\mathrm{J}$ Ocul Biol Dis Info. 2009;2:235-42.

6. Karamichos D, Zieske JD, Sejersen H, Sarker-Nag A, Asara JM, Hjortdal J. Tear metabolite changes in keratoconus. Exp Eye Res. 2015;132:1-8.

7. Khaled ML, Helwa I, Drewry M, Seremwe M, Estes A, Liu Y. Molecular and histopathological changes associated with keratoconus. Biomed Res Int. 2017;2017:16.

8. Bykhovskaya Y, Margines B, Rabinowitz YS. Genetics in keratoconus: where are we? Eye Vis. 2016;3:16. 
9. Roy AS, Shetty R, Kummelil MK. Keratoconus: a biomechanical perspective on loss of corneal stiffness. Indian $\mathrm{J}$ Ophthalmol. 2013;61:392-3.

10. Fontana L, Parente G, Tassinari G. Clinical outcomes after deep anterior lamellar keratoplasty using the big-bubble technique in patients with keratoconus. Am J Ophthalmol. 2007;143:117-24.

11. Iseli HP, Thiel MA, Hafezi F, Kampmeier J, Seiler T. Ultraviolet A/riboflavin corneal cross-linking for infectious keratitis associated with corneal melts. Cornea. 2008;27:590-4.

12. Jankov IiMR, Jovanovic V, Nikolic L, Lake JC, Kymionis G, Coskunseven E. Corneal collagen cross-linking. Middle East Afr J Ophthalmol. 2010;17:21-7.

13. Raiskup F, Spoerl E. Corneal crosslinking with riboflavin and ultraviolet A. I. Principles. Ocul Surf. 2013;11:65-74.

14. Sporl E, Huhle M, Kasper M, Seiler T. [Increased rigidity of the cornea caused by intrastromal cross-linking]. Ophthalmologe. 1997;94:902-6.

15. Wollensak G, Spoerl E, Seiler T. Stress-strain measurements of human and porcine corneas after riboflavin-ultraviolet-A-induced cross-linking. J Cataract Refract Surg. 2003;29:1780-5.

16. Raiskup-Wolf F, Hoyer A, Spoerl E, Pillunat LE. Collagen crosslinking with riboflavin and ultraviolet-A light in keratoconus: Long-term results. J Cataract Refract Surg. 2008;34:796-801.

17. Wittig-Silva C, Chan E, Islam FMA, Wu T, Whiting M, Snibson GR. A randomized, controlled trial of corneal collagen crosslinking in progressive keratoconus: three-year results. Ophthalmology. 2014;121:812-21.

18. Raiskup F, Theuring A, Pillunat LE, Spoerl E. Corneal collagen crosslinking with riboflavin and ultraviolet-A light in progressive keratoconus: ten-year results. J Cataract Refract Surg. 2015;41:41-6.

19. Liu Y, Liu Y, Zhang YN, Li AP, Zhang J, Liang QF, et al. Systematic review and meta-analysis comparing modified crosslinking and standard cross-linking for progressive keratoconus. Int J Ophthalmol. 2017;10:1419-29.

20. Hayes S, Boote C, Kamma-Lorger CS, Rajan MS, Harris J, Dooley E, et al. Riboflavin/UVA collagen cross-linking-induced changes in normal and keratoconus corneal stroma. PLoS ONE. 2011;6:e22405

21. Karamichos D, Hjortdal J, Hutcheon A, Asara J, Zieske J. Novel proteins and metabolites for the identification of keratoconus disease. Invest Ophthalmol Vis Sci. 2013;54:4071.

22. McKay TB, Sarker-Nag A, Lyon D, Asara JM, Karamichos D. Quercetin modulates keratoconus metabolism in vitro. Cell Biochem Funct. 2015;33:341-50.

23. McKay TB, Hjortdal J, Sejersen H, Karamichos D. Differential effects of hormones on cellular metabolism in keratoconus in vitro. Sci Rep. 2017;7:42896.

24. Karamichos D, Hutcheon AE, Rich CB, Trinkaus-Randall V, Asara JM, Zieske JD. In vitro model suggests oxidative stress involved in keratoconus disease. Sci Rep. 2014;4:4608.

25. Ren R, Hutcheon AEK, Guo XQ, Saeidi N, Melotti SA, Ruberti $\mathrm{JW}$, et al. Human primary corneal fibroblasts synthesize and deposit proteoglycans in long-term 3-D cultures. Dev Dyn. 2008;237:2705-15.

26. Karamichos D, Zareian R, Guo X, Hutcheon AEK, Ruberti JW, Zieske JD. Novel in vitro model for keratoconus disease. J Funct Biomater. 2012;3:760-75.

27. Sharif R, Hjortdal J, Sejersen H, Frank G, Karamichos D. Human in vitro model reveals the effects of collagen cross-linking on keratoconus pathogenesis. Sci Rep. 2017;7:12517.
28. Yuan M, Breitkopf SB, Yang X, Asara JM. A positive/negative ion-switching, targeted mass spectrometry-based metabolomics platform for bodily fluids, cells, and fresh and fixed tissue. Nat Protoc. 2012;7:872-81.

29. McKay TB, Hjortdal J, Sejersen H, Asara JM, Wu J, Karamichos D. Endocrine and metabolic pathways linked to keratoconus: implications for the role of hormones in the stromal microenvironment. Sci Rep. 2016;6:25534.

30. Wojcik KA, Kaminska A, Blasiak J, Szaflik J, Szaflik JP. Oxidative stress in the pathogenesis of keratoconus and fuchs endothelial corneal dystrophy. Int $\mathrm{J}$ Mol Sci. 2013;14:19294-308.

31. Brubaker RF, Bourne WM, Bachman LA, McLaren JW. Ascorbic acid content of human corneal epithelium. Invest Ophthalmol Vis Sci. 2000;41:1681-3.

32. Umapathy A, Donaldson P, Lim J. Antioxidant delivery pathways in the anterior eye. Biomed Res Int. 2013;2013:207250.

33. Venkata SJA, Narayanasamy A, Srinivasan V, Iyer GK, Sivaramakrishnan R, Subramanian M, et al. Tear ascorbic acid levels and the total antioxidant status in contact lens wearers: a pilot study. Indian J Ophthalmol. 2009;57:289-92.

34. Cejka C, Cejkova J. Oxidative stress to the cornea, changes in corneal optical properties, and advances in treatment of corneal oxidative injuries. Oxid Med Cell Longev. 2015;2015:10.

35. Chaerkady R, Shao H, Scott SG, Pandey A, Jun AS, Chakravarti $\mathrm{S}$. The keratoconus corneal proteome: loss of epithelial integrity and stromal degeneration. J Proteomics. 2013;87:122-31.

36. Chen Y, Mehta G, Vasiliou V. Antioxidant defenses in the ocular surface. Ocul Surf. 2009;7:176-85.

37. Cabrera MP, Chihuailaf RH. Antioxidants and the integrity of ocular tissues. Vet Med Int. 2011;2011:905153

38. Marchitti SA, Chen Y, Thompson DC, Vasiliou V. Ultraviolet radiation: cellular antioxidant response and the role of ocular aldehyde dehydrogenase enzymes. Eye Contact Lens. 2011;37:206-13.

39. Yamauchi M, Sricholpech M. Lysine post-translational modifications of collagen. Essays Biochem. 2012;52:113-33.

40. Miao L, Nielsen M, Thewalt J, Ipsen JH, Bloom M, Zuckermann $\mathrm{MJ}$, et al. From lanosterol to cholesterol: structural evolution and differential effects on lipid bilayers. Biophys J. 2002;82:1429-44.

41. Arnal E, Peris-Martinez C, Menezo JL, Johnsen-Soriano S, Romero FJ. Oxidative stress in keratoconus? Invest Ophthalmol Vis Sci. 2011;52:8592-7.

42. Shetty R, Sharma A, Pahuja N, Chevour P, Padmajan N, Dhamodaran $\mathrm{K}$, et al. Oxidative stress induces dysregulated autophagy in corneal epithelium of keratoconus patients. PLoS ONE. 2017; 12:e0184628.

43. Buddi R, Lin B, Atilano SR, Zorapapel NC, Kenney MC, Brown DJ. Evidence of oxidative stress in human corneal diseases. J Histochem Cytochem. 2002;50:341-51.

44. Kenney MC, Chwa M, Atilano SR, Tran A, Carballo M, Saghizadeh M, et al. Increased levels of catalase and cathepsin V/L2 but decreased TIMP-1 in keratoconus corneas: evidence that oxidative stress plays a role in this disorder. Invest Ophthalmol Vis Sci. 2005;46:823-32.

45. Jezek P, Hlavata L. Mitochondria in homeostasis of reactive oxygen species in cell, tissues, and organism. Int J Biochem Cell Biol. 2005;37:2478-503.

46. Mohanty K, Dada R, Dada T. Neurodegenerative eye disorders: role of mitochondrial dynamics and genomics. Asia Pac J Ophthalmol (Phila). 2016;5:293-9. 